\title{
Material Fatigue Research for Zirconia Ceramic Dental Implant: a Comparative Laboratory and Simulation Study in Dentistry
}

\author{
Ö. KARAÇALI \\ Department of Mechanical Engineering, Faculty of Engineering, \\ Istanbul University, Avcilar, Istanbul, Turkey, Turkey
}

\begin{abstract}
This research focuses on the biocompatible zirconia ceramic $\left(\mathrm{ZrO}_{2}\right)$ material and load behavior causing fatigue on the surface of the dental implant in dentistry. Fatigue fracture and wear have been identified as some of the major problems associated with implant loosening, stress-shielding and ultimate implant failure. A static and cyclic fatigue testing of ceramic dental implant in laboratory conditions for this investigation were carried out according to the ISO protocol 14801 under worst-case conditions. A finite element analysis (FEA) of dental implant with accurate geometry and material properties were developed to make realistic investigations on biocompatibility of the implant biomaterial properties and mechanical fatigue behavior of new dental implant comparing Von-Mises criteria and maximum stress levels. The comparison of calculated fatigue life simulation data of and experimental data for the biocompatible zirconia ceramic dental implants is presented. As a conclusion zirconium implant exceeded the established values for maximum incisal bite forces reported in the literature and and also shows better performance than titanium implant material. The results of fatigue of biocompatible zirconia analysis are helpful for implant biomaterial selection and design for clinical interest in dentistry.
\end{abstract}

DOI: $10.12693 /$ APhysPolA.127.1195

PACS: 81.40.Np, 87.10.Kn, 81.05.Mh, 81.70.Bt

\section{Introduction}

Today, there is scientific evidence that biomaterial zirconia dental implants integrate well and offer many advantages over titanium implants in dentistry [1]. Because of its excellent biomechanical characteristics, biocompatibility, and bright tooth-like color, zirconia (zirconium dioxide, $\mathrm{ZrO}_{2}$ ) with tetragonal crystal structure has the potential to become a substitute for titanium as dental implant material [2]. The zirconia ceramics have been employed for dental implant materials, as artificial dental roots, undergo cyclic loading for a long period in corrosive environment of mouth [3]. The ever increasing demand for higher efficiency and light weight typical of dental implants calls for maximum exploitation of a material's properties [4]. The models that are closely related to the microstructural processes provide a more reliable basis for life prediction, provided that the relevant microstructural damage mechanisms are accurately accounted for [3]. Therefore there is increasing interest in fatigue tests with biocompatible material of $\mathrm{ZrO}_{2}$.

Titanium dental implants have disadvantages like corrosion, radiating ions to the human body as well as having many separate parts to be assembled. New zirconia implants are produced in one piece. This new type biocompatible material like zirconia ceramics need to be analyzed in whole part since the hostile loads effect the

e-mail: ozdogank@istanbul.edu.tr complete body of the dental implant. New type zirconia implants are produced in one piece as shown in Fig. 1.

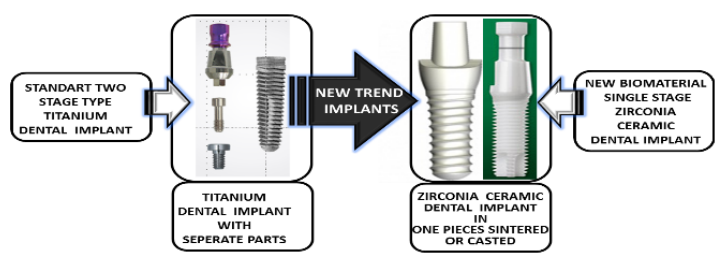

Fig. 1. Transformation into real ceramic material implant.

It is quintessential to understand the fundamentals and the material of the implant before introducing any new design modifications. Zirconia ceramics $\left(\mathrm{ZrO}_{2}\right)$ material properties given in Fig. 2. A need for non-metallic restorative materials with optimal aesthetics and characteristics such as biocompatibility, color stability, high wear resistance and low thermal conductivity are often forwarded as reasons for the use of ceramics in dentistry [4]. Ceramics are, however, brittle materials because of atomic bonds that do not allow the atomic planes to slide apart when subjected to load as depicted in Fig. 3. Thus, ceramics cannot withstand deformation without fracturing [2]. Several studies have indicated that flexural strength values of $1200 \mathrm{MPa}$ and fracture toughness values of $9 \mathrm{MPa}$, which are possible with zirconia, are substantially higher than for other ceramics and that this material therefore could be used for highly loaded, all-ceramic restorations.

The ceramics meet the demands of the application requirements along with the biological criteria of compati- 


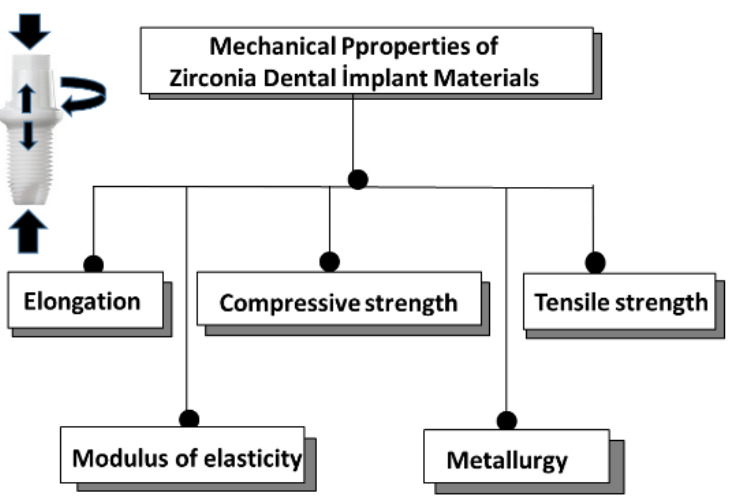

Fig. 2. Zirconia material implants mechanical properties.

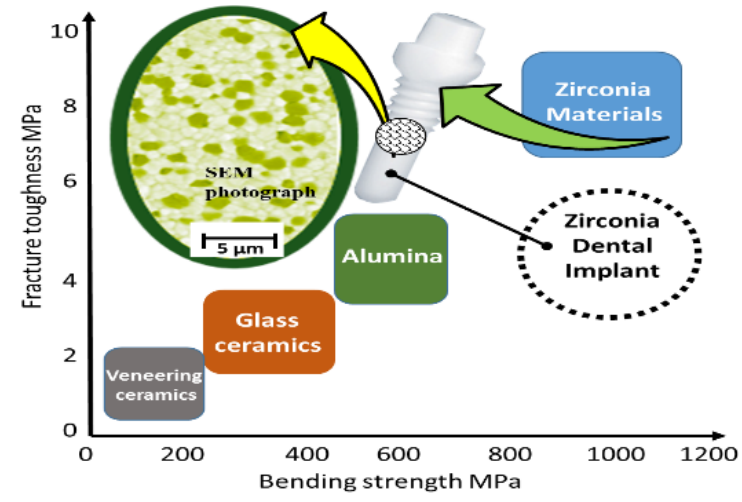

Fig. 3. Fracture strength of different ceramic materials.

bility and inertness/resorb ability. The tailoring of composition, microstructure, physical properties and molecular surface chemistry of various bio ceramic implants are the pillars of the continuing success of these materials that offer the chance of a damage free, pain free, maintenance free stable life for a longer time. A special indication of the ceramic Implant System is recommended for patients with metal allergies and chronic illnesses due to metal allergies [1]. Fig. 4 shows a schematic picture of the cross-section of a deformed ceramic biomaterial surface, surrounded in a physiological environment.

The aim of the present study therefore was to evaluate the strength of one zirconia and of one crown system in a standardized way by mimicking the clinical situation with cyclic mechanical pre-loading under the null-hypothesis that there is not any difference between the fracture resistances between crowns with a core of zirconia.

Finite element analysis (FEA) model was developed to understand the value of testing to validate models, improve production, and increase the overall quality of parts. The time and expense of testing might seem like an easy place to reduce costs, but unforeseen failures after parts are already in service are much more difficult to bear. The proposed research comprised fatigue test of zirconia dental implant material is to establish long-term durability in laboratory conditions.

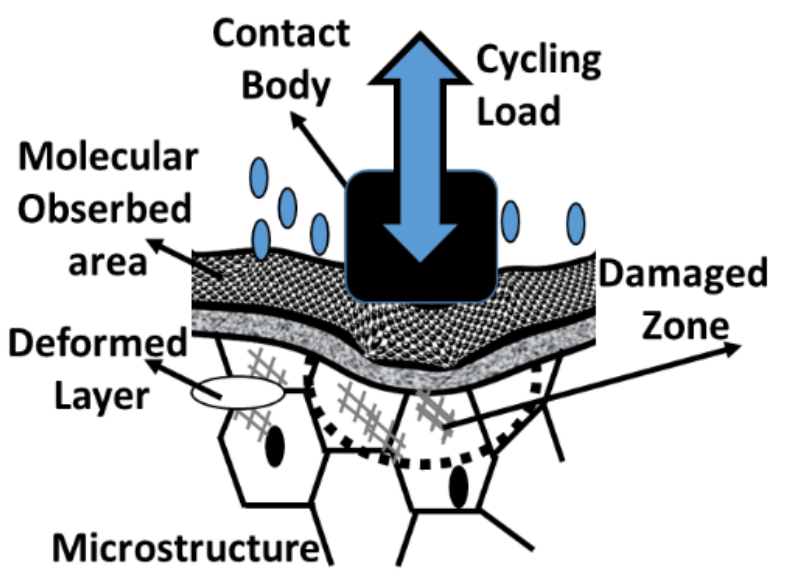

Fig. 4. Deformed ceramic surface interactions.

Fatigue testing can be performed at a various levels of development. Standard methods can be utilized when the raw material is tested, since specimens can be machined to standard configurations. For mechanical testing, single load to fracture was performed by 6027 Instron fatigue test machine in laboratory and the mean values were calculated for the maximum load $\left(F_{\max }\right)$ as shown in Fig. 5.

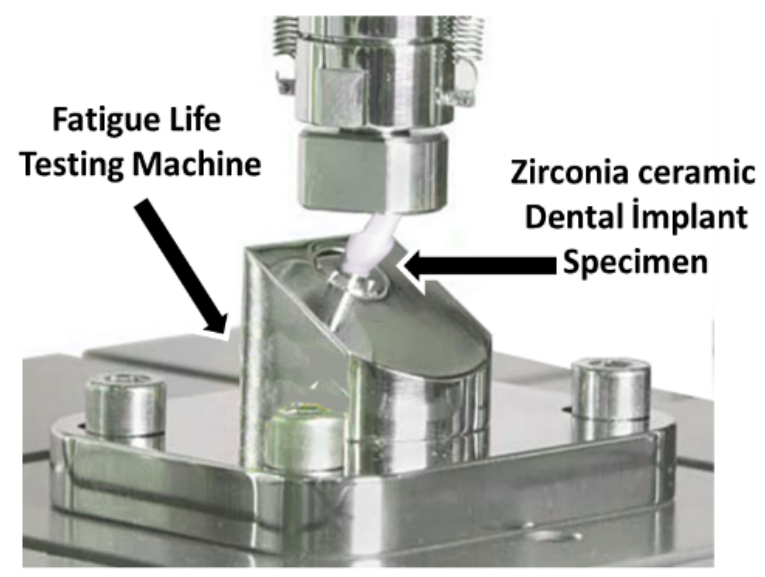

Fig. 5. Fatigue test machine in laboratory.

\section{Material and method}

When a material is subjected to a cyclic load for a long time, failure might occur at stress levels much lower than failure stress under a static load. Failure of materials under cyclic load is called fatigue failure. The objectives of the research from the fatigue and fracture point of view in this paper was conceived in three steps: (1) a fatigue test environment was developed and a test was realized to analyze the behavior of the ceramic dental implant (2) a FEA model was developed to determine theoretical stress levels and locations for comparison of stress-strain and to validate mechanical testing of ceramic dental implants (3) stress/life (S-N) curve approach for fatigue-wear was used for simulation of physiologic multi-axial loading. 
To test the mechanical properties of the zirconia implant, fatigue strength testing according to the ISO 14801 "Dentistry-Fatigue test for endosseous dental implants" standard was carried out, simulating the functional loading of an endosseous dental implant body and its abutment components under worst-case conditions in a laboratory study. Cyclic loading tests were carried out by means of a servo hydraulic dynamic testing machine at loads between 100 and $450 \mathrm{~N}$ with 5 million validation cycles at $15 \mathrm{~Hz}$ at the same benchmark load as the ceramic implants, the implant met all acceptance criteria. The angle of loading force was at $30^{\circ}$ to the axis of the implant body as depicted in Fig. 6 .

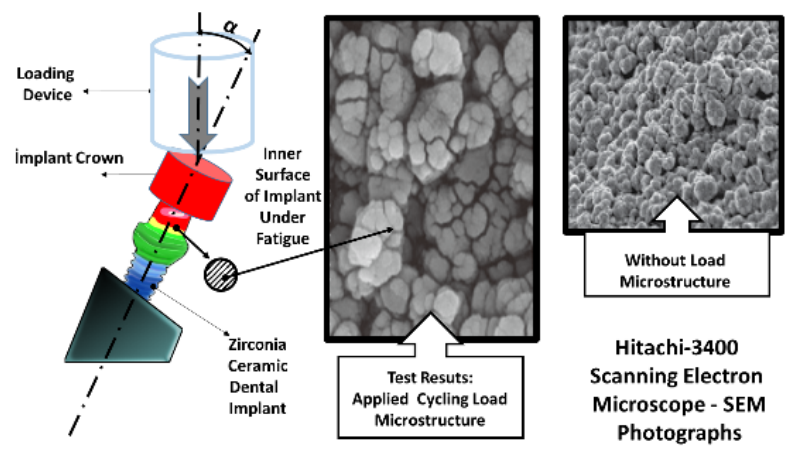

Fig. 6. Fatigue testing of $\alpha=30^{\circ}$ off-axis and microstructure.

The load was applied via a stainless steel rod (pinloaded using a small center drill point) to withstand external forces and to prevent the rod and the attached holding fixture to deflect too far laterally. This test result show that the mechanical properties offered by implant indicated for teeth has an excellent mechanical properties even in the most severe conditions. The simulation of 5 million cycles is to simulate an oral environment function of about 15 years. Zirconia has a flexural strength and fracture toughness almost twice as high as that of alumina, which makes zirconia very resistant to masticatory forces, while still maintaining an exact precision. These proven mechanical qualities allow the restorative dental implants high flexibility for indication and application. This resulted in a combination of compression, bending, and shear loads in the device. The tests were performed both statically, for single overload conditions, and in repeated loading, to provide fatigue curves of load versus cycles required to cause failure. Hitachi-3400 scanning electron microscope (SEM) was employed to examine the surface and elementally analyze the zirconia microstructure after cyclic loading fatigue test as shown in Fig. 6. The image showed no obvious fracture surfaces that may be attributed to the fatigue process.

ANSYS Workbench 14.0 was used to generate a threedimensional mesh of a model created in SOLIDWORKS software with the actual size specifications. In Fig. 7, stress plots of zirconia implant is shown by FEA. Dental implant model, an average of 92,000 nodes and 52,000 elements were used. FEA was carried out by comparing
von-Mises and maximum stress levels obtained from the calculation as in Fig. 7.

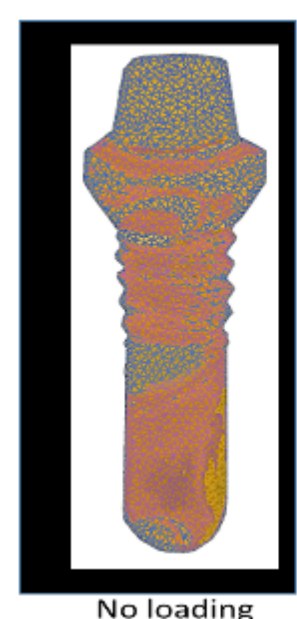

No loading

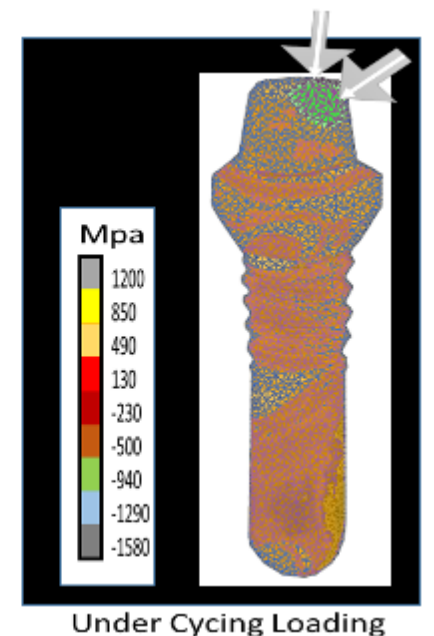

Under Cycing Loading
Fig. 7. Stress plots of zirconia implant by FEA.

To validate the credibility of calculated fatigue life, actual prototypes were built with the design specifications and tested using FEA. The main advantages of performed computer simulations are that it is fast, efficient and cheap. A comparison of the calculated fatigue life with experimental fatigue life data displayed the accuracy and reliability of the computer simulation method. This simulation's purpose is to verify that the ceramic part of the proposed design is strong enough to achieve the fatigue life $\left(5 \times 10^{6}\right.$ Cycle) suggested by the (ISO) - International Organization for Standardization. ISO provides more specific specifications and constraints in ISO 14801Fatigue test for endosseous dental implants. These specifications and constraints shown in Fig. 6 were strictly followed for both simulation and testing. The endosseous dental implant is clamped at a distance $3.0 \pm 0.1 \mathrm{~mm}$ apically from the nominal bone level and such that it makes a $30^{\circ} \pm 1^{\circ}$ angle with the loading direction of the testing machine. Simulation is carried out with a unidirectional load with a frequency less than $15 \mathrm{~Hz}$. ANSYS Workbench was used along with $\mathrm{S}-\mathrm{N}$ curve to estimate the fatigue life in this research.

Engineering fatigue data was presented in the form of an $\mathrm{S}-\mathrm{N}$ curve where stress $(\mathrm{S})$ is plotted against the number of cycles to failure $(\mathrm{N})$. This data was useful for lifetime prediction analysis of ceramic dental implant. As the stress decreases, the ceramic endures a greater number of cycles; below a certain stress, the S-N curve levels off and becomes horizontal at a particular stress level. Figure 8 shows the S-N curve of the as-cast $\left(\mathrm{ZrO}_{2}\right)$ alloy, and the results of fatigue tests from the current study. The first-cycle tests of the specimens show comparable fatigue life with one sample on the left side of the S-N curve and two samples on the right side of the S-N curve (colored). This stress level is known as the fatigue limit. Below this limit, the ceramics could theoretically endure an infinite number of cycles without failure. 


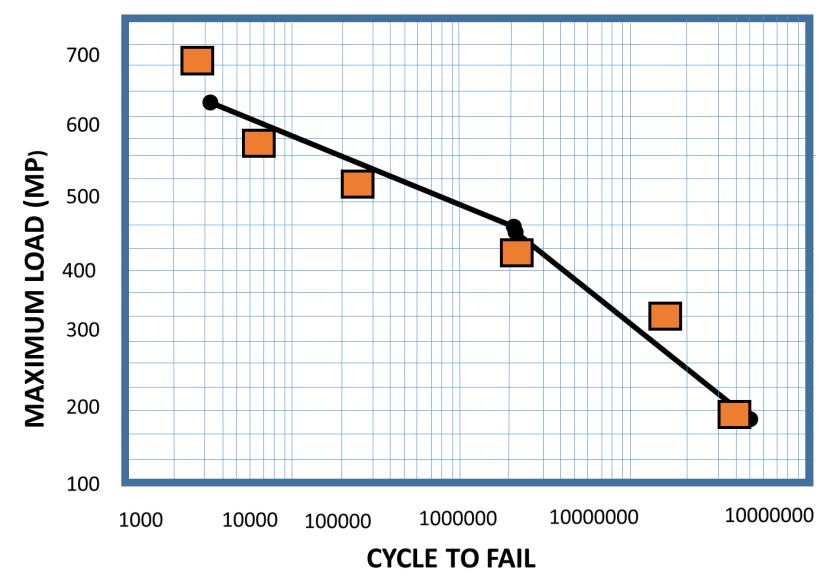

Fig. 8. Comparison of calculated fatigue life simulation data and experimental data.

\section{Conclusions}

This laboratory and simulation study aimed to provide comparative data on the mechanical performance of zirconia ceramic implants in dentistry. One of the vital factors in the ceramic dental implant design process is to investigate the fatigue behaviors. In this research, a three-dimensional finite element analysis model was constructed to investigate the fatigue behaviors of new ceramic dental implant design and fatigue test was done in order to prove the reliability of proposed design in dentistry. Scientific studies about maximum bite forces with natural teeth in several materials have been found, which have observed values between $60 \mathrm{~N}$ and $645 \mathrm{~N}$ for the maximum bite force with molar teeth. The zirconiumceramic implants investigated in the present study exhibited a maximum fracture strength of $672 \mathrm{~N}$ during static loading, and $475 \mathrm{~N}$ at 800,000 to 5 million cycles run out point and $403 \mathrm{~N}$ at 10,000 cycles run out point during cyclic loading. From the load and stress values point of view, the FEA were mostly consistent with the physical test results.

It was shown that computational modeling and 3D simulation using finite element analysis enabled the realistic prediction of ceramic dental implant fatigue behavior. The main advantage of performed computer simulations is efficient, faster and cheaper than real experimental work. A comparison of the calculated fatigue life with experimental fatigue life data displayed the accuracy and reliability of the computer simulation method in dentistry.

\section{Acknowledgments}

This research was supported by Scientific Research Projects (BAP) of Istanbul University, Turkey, Project No: 46540 . The support of Istanbul University is greatly appreciated.

\section{References}

[1] D.R. Prithviraj, K.M. Regish, S. Deeksha, D.P. Shruthi, J. of Clin. Exp. Dent. 3, 240 (3).
[2] E. Mick, J. Markhoff, A. Mitrovic, A. Jonitz, R. Bader, J. of Mat. 6, 4001 (2013).

[3] P.G. Coelho, N.R. Silva, E.A. Bonfante, P.C. Guess, E.D. Rekow, V.P. Thompson, J. of dent. mat. 25, 1122 (2009).

[4] A. Merdji, B. Bouiadjra, T. Achour, B. Serier, B.O. Chikh, Z.O. Feng, Comp. Mat. Science. 49, 126 (2010). 